\title{
Idiopathic Pulmonary Fibrosis: Clinical Behavior, Pathogenic Mechanisms, and Therapeutic Approach
}

\section{Moisés Selman ${ }^{1}$ and Annie Pardo ${ }^{2}$}

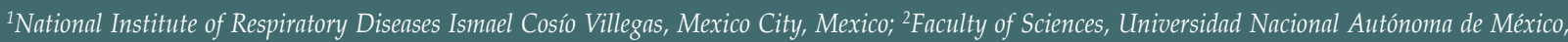
Mexico City, Mexico

\section{ABSTRACT}

Idiopathic pulmonary fibrosis is a chronic, progressive, and usually lethal lung disease of unknown origin with a median survival time of 3-5 years after diagnosis. Although the pathogenic mechanisms and the sequence of the events leading to idiopathic pulmonary fibrosis have not been completely elucidated, a growing body of evidence indicates that the disease is caused by persistent epithelial injury and activation in genetically susceptible older individuals, with a signature of epigenetic changes that provokes the expansion of the fibroblast population and its differentiation to myofibroblasts, which secrete exaggerated amounts of extracellular matrix with the subsequent architectural remodeling and lung destruction. In this review, we discuss the clinical diagnosis and behavior of idiopathic pulmonary fibrosis and highlight the role of the genetic architecture, epigenetic modifications, and aging in the pathogenesis of the disease. Finally, we discuss the current therapeutic approaches, including the use of nintedanib and pirfenidone according to recently completed phase III idiopathic pulmonary fibrosis clinical trials. (BRN Rev. 2015;1:13-25) Corresponding author: Moisés Selman,mselmanl@yahoo.com.mx; moises.selma@salud.gob.mx

Key words: Aging. Epigenetic. Genome-wide association study. IPF. Pulmonary fibrosis. 
Idiopathic pulmonary fibrosis (IPF) is a specific form of chronic, fibrosing interstitial pneumonia of unknown etiology associated with the histopathological pattern of usual interstitial pneumonia $(\mathrm{UIP})^{1,2}$. The disease is usually progressive and irreversible, leading to a gradual but relentless destruction of the lung parenchyma, showing a mean survival of approximately 3-5 years from the time of diagnosis.

\section{DIAGNOSIS AND CLINICAL BEHAVIOR}

Idiopathic pulmonary fibrosis affects primarily middle-aged and elderly adults, mainly former or current smokers, and in this context its diagnosis should be considered in any individual over 50 years old (mainly ever smoker) with unexplained and slowly progressive dyspnea, or inexplicable and prolonged (months) cough. Unfortunately, IPF is often misdiagnosed because the early manifestations of the disease are nonspecific, and it is incorrectly diagnosed as asthma, chronic obstructive pulmonary disease (COPD), emphysema, or heart disease, leading to considerable delay between the beginning of symptoms and the precise diagnosis. Moreover, the incorrect diagnosis is often associated with the use of ineffective or potentially harmful treatment and delays the opportunity for possible lung transplant, increasing the risk of death ${ }^{3}$.

The correct diagnosis of IPF requires exclusion of other known causes of interstitial lung disease (e.g. environmental exposures, autoimmune diseases, and drug toxicity) and the presence of a pattern of UIP on high-resolution computed tomography (HRCT) in patients not undergoing surgical lung biopsy, or specific combinations of HRCT and surgical lung biopsy patterns in patients undergoing surgical lung biopsy ${ }^{1}$ (Fig. 1). However, diagnosis is challenging and the latest international guidelines emphasize the importance of a multidisciplinary team in the initial diagnostic approach of patients with suspected IPF. This team should include a pulmonologist, a radiologist, and a pathologist, with further contribution from a rheumatologist and an expert in occupational medicine when appropriate ${ }^{1}$.

The natural history of IPF is highly variable and the course of disease in an individual patient is difficult to predict. Most patients progress slowly or even remain stable for some time, but some patients deteriorate rapidly, showing an accelerated course of the disease $\mathrm{d}^{2,4}$. In addition, around $10 \%$ of patients present unexpected deterioration with a sudden and acute worsening of symptoms and lung function ${ }^{5}$. These episodes, when occurring without an identifiable cause, are known as acute exacerbations and are often fatal.

\section{PATHOGENESIS}

Idiopathic pulmonary fibrosis is a multifactorial disease that likely occurs as a result of the interaction between some environmental factors and genetic components. However, the pathogenic mechanisms as well as the sequence of cellular and molecular abnormalities leading to IPF have not been elucidated. Recently, we proposed that the convergence of three "IPF-facilitator" processes are essential for the development of this disease ${ }^{6}$ : (i) genetic architecture, (ii) aging, and (iii) epigenetic changes, likely associated to environmental factors and to aging (Fig. 2). 

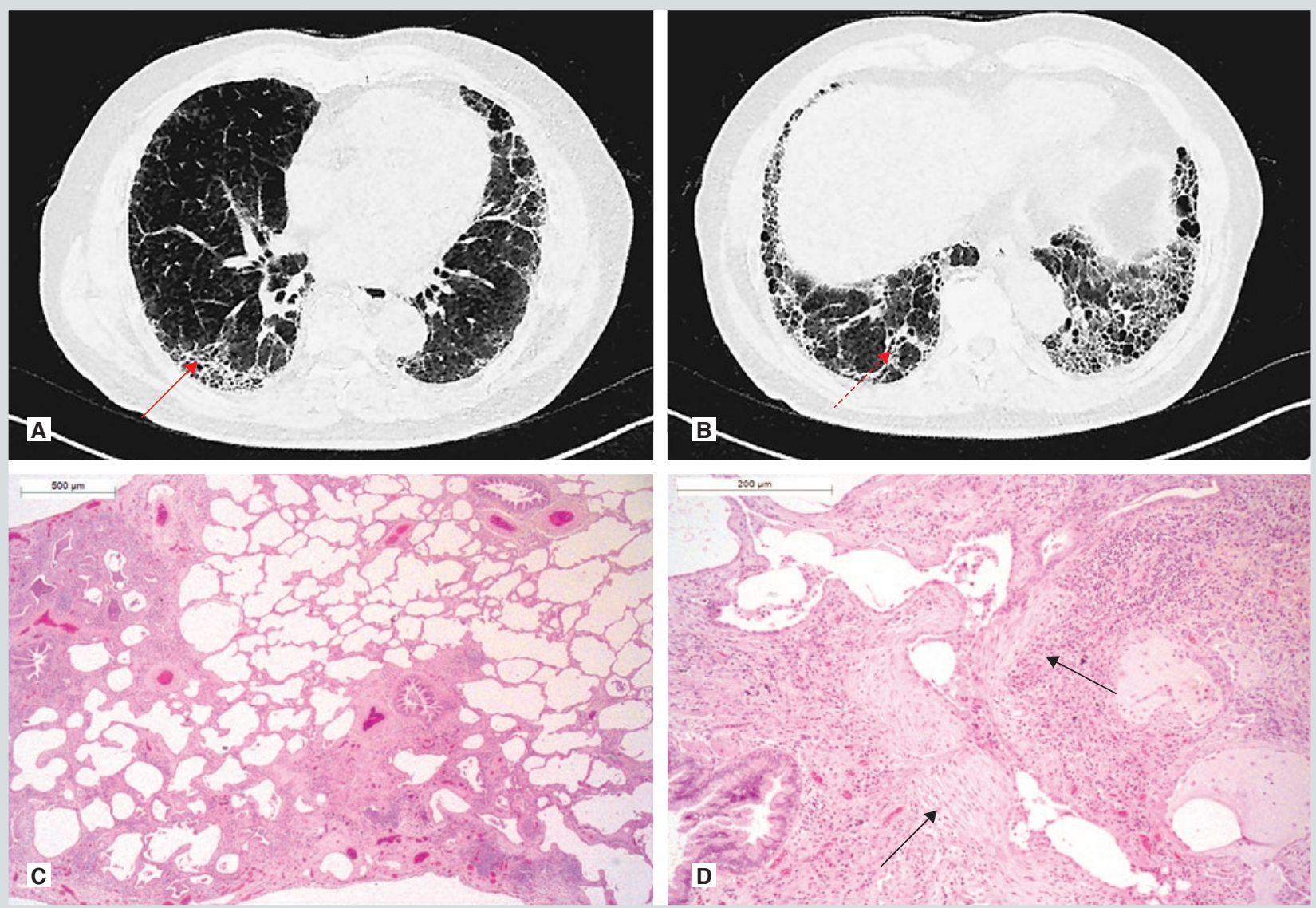

Figure 1. A and B: two HRCT images representing a usual interstitial pneumonia pattern. Distinctive imaging features include the presence of honeycombing (red arrow), traction bronchiectasis (black dotted arrows) and a reticular pattern. Lesions are typically located along sub-pleural regions. C and D: histopathologic characteristics of a usual interstitial pneumonia pattern. C: low-power view shows marked fibrosis with architectural distortion, honeycombing in a predominantly sub-pleural distribution close to uninvolved (almost) normal lung (hematoxylin-eosin, original magnification $\times 20$ ). D: high-power view shows fibroblast foci (black arrows) at the edge of dense fibrosis and honeycomb lesions (hematoxylin-eosin 40x).

\section{The genetic architecture of idiopathic pulmonary fibrosis}

The genetic risk factors associated to susceptibility for the development of sporadic IPF are uncertain, although it is well known that mutations, primarily in some components of telomerase, strongly contribute to the development of familial $\mathrm{IPF}^{7}$. Familial IPF comprises around $5-10 \%$ of all cases, and the majority of lineages demonstrate an autosomal-dominant pattern of inheritance with reduced penetrance. However, the gene mutations involved have been revealed only in $20-25 \%$ of the familial cases.

The underlying genetic basis of sporadic IPF has been evaluated through the candidate gene approach, linkage and fine mapping, and genome-wide association studies (GWAS). Studies focused on biologically relevant candidate genes have revealed some interesting 


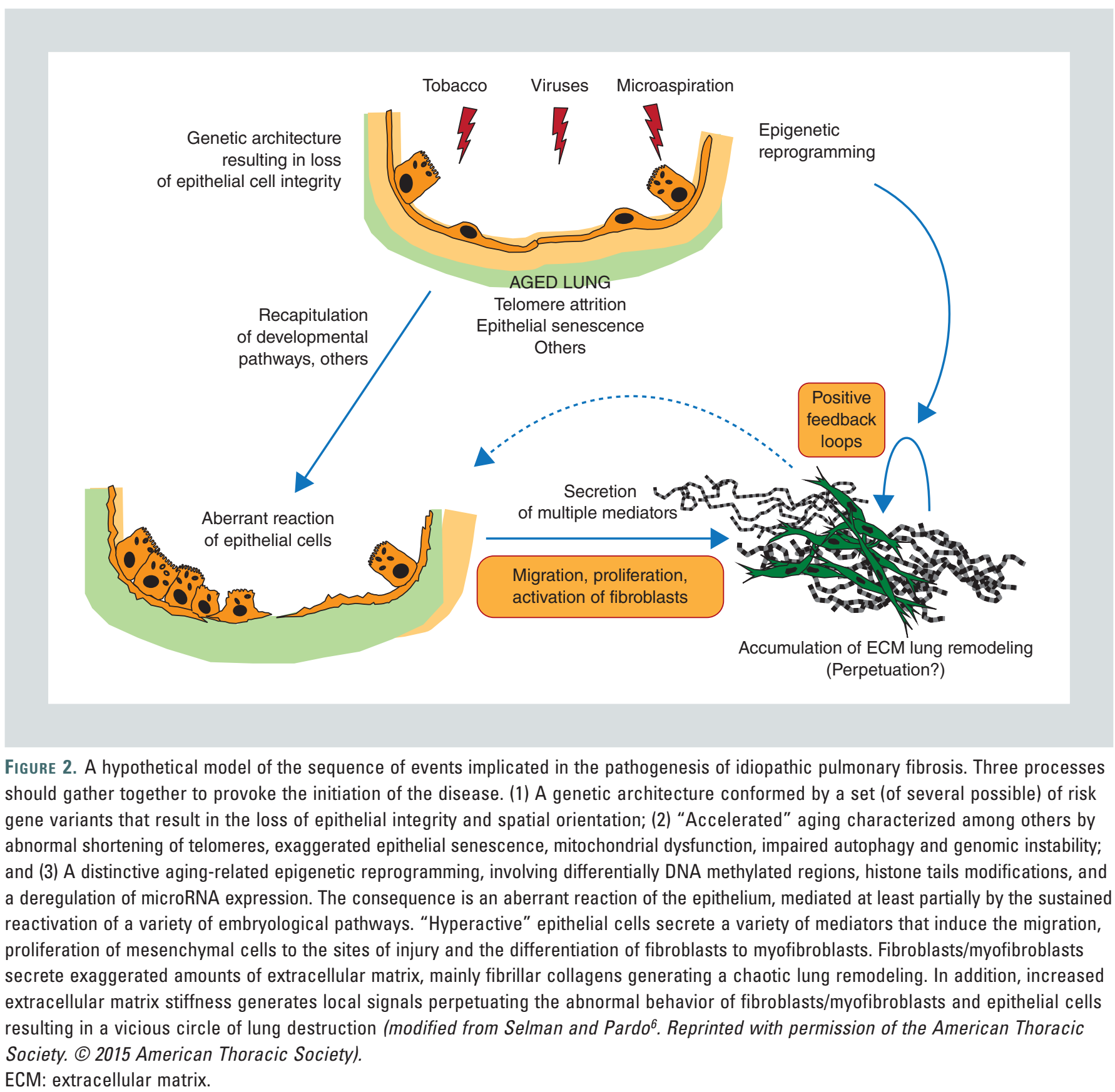

ECM: extracellular matrix.

genes, but there are only few examples where significant results have been replicated in independent cohorts ${ }^{8}$.

Linkage and fine mapping identified a region of interest on the p-terminus of chromosome 11 where it was found that the minor-allele of the single-nucleotide polymorphism (SNP) rs35705950, located $3 \mathrm{~kb}$ upstream of the MUC5B transcription start site, was present at a frequency of $34 \%$ among subjects with familial interstitial pneumonia (most of them with IPF), 38\% among subjects with sporadic IPF, and $9 \%$ among controls 9 . The odds ratios 
for disease among subjects who were heterozygous for the minor allele of this SNP were 6.8 for familial interstitial pneumonia and 9.0 for sporadic IPF, which increased several-fold in those who were homozygous9. This promoter polymorphism in MUC5B has been confirmed by multiple studies as a common risk factor with a large genetic effect on development of pulmonary fibrosis ${ }^{10,11}$. The putative role of MUC5B in the pathogenesis of the disease is presently unknown, but curiously, some studies have shown that IPF patients carrying the MUC5B variant that increases the risk to develop the disease show a significantly improved survival ${ }^{12}$.

The first GWAS approach in IPF was performed in a Japanese population and revealed a significant association with the disease of a SNP in the intron 2 of the TERT gene (rs2736100), which encodes the reverse transcriptase of telomerase $^{13}$. Subsequently, Schwartz, et al. ${ }^{14}$ performed the largest GWAS carried out to date, involving 1,616 patients with idiopathic interstitial pneumonias, mostly IPF, and 4,683 controls, with follow-up replication analyses in 876 cases and 1,890 controls. The results confirmed the association of IPF with TERT at $5 \mathrm{p} 15, M U C 5 B$ at $11 \mathrm{p} 15$ and the $3 \mathrm{q} 26$ region near TERC, and identified seven new associated loci, including family with sequence similarity 13, member A (FAM13A; 4q22), desmoplakin (DSP; 6p24), oligonucleotide-binding fold containing-1 (OBFC1; 10q24), ATPase, class VI, type 11A (ATP11A; 13q34), dipeptidyl-peptidase 9 (DPP9; 19p13), and chromosomal regions 7q22 and 15q14-15.

Almost at the same time, Noth et al..$^{15}$ reported a three-stage GWAS, with stage one being a discovery GWAS and stages two and three independent case-control studies. Their results confirmed previously reported gene variants, including MUC5B and TERT SNPs, and revealed novel variants in toll interacting protein (TOLLIP, (rs111521887, rs5743894, rs5743890; 11p15.5) and signal peptide peptidase like $2 \mathrm{C}$ (SPPL2C, rs17690703; 17q21.31) associated with IPF susceptibility. The TOLLIP is a critical regulator of toll-like receptor-mediated innate immune responses, but is also a strong inhibitor of transforming growth factor beta (TGF-b) signaling and of the epithelial to mesenchymal transition ${ }^{16}$. Also intriguingly, patients carrying the minor (protective) allele rs5743890 in the TOLLIP gene are at increased risk for mortality from IPF.

In general, all these genetic findings indicate that genes involved in cell-cell adhesion, epithelial cell integrity and regeneration, and host defense mechanisms, contribute to risk of IPF.

\section{Aging processes play a major role in the pathogenesis of idiopathic pulmonary fibrosis}

Age is the strongest demographic risk factor for IPF and actually most patients are older than 60 years at the time of diagnosis, suggesting a mechanistic link between chronological age and this disease $\mathrm{e}^{6,17}$.

In this context, several of the cellular and molecular hallmarks of aging have been found to be aggravated in IPF lungs ${ }^{18}$. For example, one common finding in aging is the accumulation of genetic damage throughout life, and microsatellite instability and loss of heterozygocity have been found in a subset of IPF patients ${ }^{19}$. Likewise, aging is associated 
with progressive and cumulative loss of telomere-protective sequences from chromosome ends, which involve the loss of the regenerative capacity. In this regard, abnormal shortening of telomeres is often found in leukocytes and epithelial cells in patients with sporadic IPF, and telomerase mutations are the main identifiable cause of familial IPF'20,21.

Another hallmark of aging is mitochondrial dysfunction ${ }^{18}$, and numerous and large dysmorphic mitochondria and age-related mitochondrial dysfunction with impaired mitophagy were recently identified in the alveolar epithelial cells from the lungs of patients with advanced $\mathrm{IPF}^{22}$. Interestingly, there is emerging evidence suggesting that mitochondrial reactive oxygen species is involved in TGF- $\beta$ signaling and expression of pro-fibrotic genes ${ }^{23}$.

Cellular senescence represents a stable arrest of the cell cycle coupled to stereotyped phenotypic changes, usually caused by telomere shortening and non-telomeric DNA damage that progressively occur with chronological aging $^{18}$. Two recent reports have demonstrated that accelerated alveolar epithelial senescence is present in the lungs of patients with IPF, evidenced by senescence-associated $\beta$-galactosidase staining and immunohistochemical detection of $\mathrm{p} 21^{24,25}$. Importantly, the senescence-associated secretory phenotype may, at least partially, explain the production of so many biological mediators secreted by the abnormally activated epithelial cells ${ }^{6}$ (Table 1).

The activities of the two principal proteolytic systems implicated in protein quality control, namely, the autophagy-lysosomal system and the ubiquitin-proteasome system, decline with aging $^{18}$. In this context, a growing body of
TABle 1. Mediators expressed by epithelial cells in idiopathic pulmonary fibrosis lungs

\section{Mediator}

Cytokines and Growth Factors

- Platelet-derived growth factor (PDGF)

- Transforming growth factor-beta (TGF-b)

- Connective-tissue growth factor (CTGF)

- Tumor necrosis factor-alpha (TNF-a)

- Osteopontin (OPN)

- Insulin-like growth factor (IGF-1)

- Insulin-like growth factor binding proteins (IGFBP) 3 and 5

- Angiotensinogen (AGT)

- Fibroblasts growth factor 9 (FGF-9)

- Neuregulin (NRG-1a)

- Endothelin 1 (ET-1)

Matrix metalloproteinases (MMPs) and their tissue inhibitors

- MMP-1

- MMP-7

- MMP-19

- MMP-14

MMP-15

- TIMP-4

Chemokines

- CCL17/thymus and activation-regulated chemokine (TARC)

- CCL2/monocyte chemotactic protein-1

- CXCL12

Coagulation factors

- TF/FVIIa/FX ternary complex

- Plasminogen activator inhibitor-1

- Protease-activated receptor-1 and-2

Developmental Pathways

- Wnt-pathway components

- Sonic Hedgehog

Others mediators

- Dimethylarginine dimethylaminohydrolase (DDAH)

- Autotaxin

- Sphingosine-1-phosphate

Pigment epithelium-derived factor

- Hypoxia-inducible factor-1a (HIF-1a)

- Heparan sulfate 6-0-endosulfatase 2 
evidence has demonstrated the presence of endoplasmic reticulum stress and unfolded protein response in alveolar epithelial cells in lungs of patients with sporadic and familial $\mathrm{IPF}^{26}$. Likewise, recent studies indicate that autophagic activity is diminished in IPF lungs ${ }^{25,27}$.

Finally, the decline in the regenerative potential (stem cell exhaustion) of tissues is another characteristic of aging, but studies in IPF are scant.

\section{The epigenetic contribution to idiopathic pulmonary fibrosis pathogenesis}

The term "epigenetics" refers to heritable changes in phenotype or gene expression that cannot be directly attributed to changes in DNA sequence. Epigenetic changes involve primarily DNA methylation, histone modifications, and non-coding RNA-mediated, all of which control chromatin architecture and gene transcription and, therefore, cell fate, biological processes, and broader phenotypes. Although stable enough to maintain a cellular state and identity, epigenome is dynamic enough to respond to environmental signals.

\section{DNA methylation}

DNA methylation involves the addition of a methyl group to the $5^{\prime}$ position of cytosine in CpG dinucleotides usually in islands and island shores. The methyl groups promote a conformational change of DNA structure and, as a consequence, the transcription factors cannot recognize the DNA, resulting in repression of transcription. Importantly, with aging there is a gradual and stochastic process of modifications in DNA methylation through random errors that are bidirectional, i.e. include hypo-and hypermethylations. This phenomenon, known as "epigenetic drift", is more evident in rapidly proliferating cells, which may be more vulnerable to this process due to an increased rate of mitotic divisions and weaker fidelity in transferring the methylation marks ${ }^{28}$. Also relevant, the rate of epigenetic drift can vary by cell type and between individuals and is likely impacted by both endogenous and exogenous factors. As such, the epigenetic drift may contribute to the age-associated increased risk for development of IPF.

Global methylation and gene expression have been recently examined in IPF lungs. In the latest report, Yang, et al., using comprehensive high-throughput arrays for relative methylation methodology, examined 4.6 million CpG sites distributed across the human genome, and simultaneously, the gene expression changes in lung tissues from 94 IPF patients and 67 control subjects ${ }^{29}$. They found that the IPF lungs have a large number of disease-associated methylation changes that also affect gene expression in the opposite direction. Methylation changes were predominantly located in gene bodies and CpG island shores. Functional analyses identified several biologically relevant methylation-expression relationships in IPF lung. Among the most enriched canonical pathways were several that have been implicated in the pathogenesis of IPF, for example CXCR4 signaling, thrombin signaling, Wnt/ $\beta$-catenin signaling, and epithelial adherens junction signaling. Analysis of binding motifs in promoters revealed overrepresentation of regulators of lung development, specifically, $\beta$-catenin, GLI1, and FOXC2. Finally, network analysis identified 10 high-scoring 
networks, the most significant centering around amyloid $\beta$ precursor (APP) protein that contains the transcription factor CASZ1. The APP is expressed in the lungs and has been shown to bind directly to TGF- $\beta 1^{30}$. The rest of the networks contain genes related to development (FZD8, GLI3, JAG2, WNT5B, WNT10A), cilium (CELSR1, FOXJ1, RFX2, RPGRIPL1), tight junctions (CTNND2, SLDN5, SLDN11, TJP2), as well as those with IPF-associated genetic polymorphisms mentioned above (DSP, TOLLIP). Taken together, this study suggests that several biologically relevant methylation-expression modifications may contribute to the development of IPF.

\section{Histone modifications and chromatin remodeling}

Histone tails are subject to multiple post-translational modifications such as phosphorylation, methylation, acetylation, and ubiquitination ${ }^{31}$. The combination of these distinct covalent modifications constitute the "histone code" that plays a central role in modulating DNA accessibility and in that way may change the gene expression pattern.

Studies of chromatin remodeling in IPF are scant, but some biopathogically meaningful genes have been shown to be epigenetically modulated through this mechanism. For example, prostaglandin $\mathrm{E}_{2}\left(\mathrm{PGE}_{2}\right)$ a potent inhibitor of lung fibroblast proliferation and migration, as well as of collagen production, is significantly decreased in $\mathrm{IPF}^{32}$. The $\mathrm{PGE}_{2}$ is produced from endogenous arachidonic acid via the cyclooxygenase (COX) pathway. In this context, it was demonstrated that COX-2 gene transcription in IPF fibroblasts is defective due to deficient histone $\mathrm{H} 3$ and $\mathrm{H} 4$ acetylation as a result of decreased recruitment of histone acetyltransferase and increased recruitment of the several transcriptional corepressor complexes to the COX-2 promoter ${ }^{33}$. These findings indicate that defective histone acetylation contributes to the decrease of COX-2 gene transcription in IPF.

\section{Non-coding ribonucleic acids}

Non-coding RNAs play a crucial role in maintaining genomic stability, which is essential for cell behavior and survival. Non-coding regulatory RNAs can generally be divided into two major classes on the basis of their size, and include the so-called small-interfering RNAs, microRNAs, and long non-coding RNAs. To date, the studies in IPF have been focused in the putative role of microRNAs, a class of small non-coding RNAs of 22-23 nucleotides in length, which negatively regulate the expression of many different genes ${ }^{34}$. According to the current literature, $\approx 40$ microRNAs have been linked to fibrosis in various organs and disease settings ${ }^{35}$. In the context of IPF, 94 microRNAs have been found differentially expressed: 43 increased and 51 decreased. In general, microRNAs that silence profibrotic messenger RNAs have been found to be decreased, e.g. miR-17-92, miR-200, miR29, miR-26, and let7d, resulting in the increase of TGF-b signaling, and in the de-repressing of a number of fibrosis-associated genes ${ }^{35-37}$.

Likewise, IPF lungs show an increase of microRNAs that repress or degrade antifibrotic mRNAs. This is the case of miR-21 that, among others, affects Smad7, an inhibitor of TGF-b signaling, miR-199a-5p that degrades the 
mRNA of caveolin-1, and miR-96 that decreases FOXO3a, which may contribute to the apoptosis-resistance of IPF fibroblasts ${ }^{35,36}$. Interestingly, Parker, et al. found that genes encoding IPF-associated extracellular matrix (ECM) proteins are targets for miR-29, which was downregulated in fibroblasts grown on IPF-derived ECM, and basal expression of ECM targets was restored by overexpression of miR-29, suggesting a positive feedback loop between fibroblasts and aberrant $\mathrm{ECM}^{38}$.

On the other hand, some deregulated microRNAs appear to participate in the recapitulation of developmental pathways, such as the WNT/b-catenin signaling pathway, a critical process in the aberrant activation of epithelial cells and fibroblasts. Thus for example, miR-154 is increased in IPF fibroblasts, and it has been shown that transfection of fibroblasts with miR-154 caused activation of the WNT pathway. Furthermore, the use of ICG001 and XAV939, inhibitors of the WNT/ $\beta$-catenin pathway, reduced the proliferative effect of miR-154 $4^{37}$. Similarly, WNT1-inducible signaling pathway protein 1 (WISP1), which is a highly expressed profibrotic mediator in the alveolar epithelial cells of IPF lungs, is regulated by miR-92a, which is decreased in $\mathrm{IPF}^{38}$. Moreover, an inverse relationship for WISP1 and miR-92a was found in a TGF- 31 -dependent lung fibrosis model in vivo ${ }^{39}$.

\section{Putative sequence of the cellular and molecular pathogenic mechanisms}

The pathogenic mechanisms of IPF are still largely unknown. For many years the prevalent hypothesis was that chronic, unresolved alveolitis of unknown etiology led to subsequent progressive fibrosis. However, a growing body of evidence strongly support that the starting pathological event in IPF is the deregulation of epithelial cell function, whose aberrant activation results in the secretion of multiple mediators that in turn provoke the migration, proliferation, and activation of mesenchymal cells ${ }^{2}$ (Table 1).

Hypothetically, repetitive micro-injuries (or propagation of some) caused by smoking or other exposures, micro-aspiration, viral infections, etc., provoke in aging lungs of patients with a determined genetic architecture and epigenetic changes, death of some epithelial cells but the hyperactivation of others.

However, the mechanisms that operate in the aberrant activation of epithelial cells (and perhaps fibroblasts) in IPF lungs are uncertain.

A strong body of evidence indicates that the recapitulation of developmental pathways may play a role (Fig. 2). Embryological signaling networks orchestrate the biological processes and interactions, which will result in distinct tissue architectures and functions. After birth, many of these pathways are suppressed or restricted to tissue-specific stem cell maintenance, but may be re-expressed in response to tissue injury in a strictly regulated pattern favoring tissue regeneration, or deregulated contributing to adult diseases, including IPF. Thus, the analyses of transcriptional and epigenetic signatures have revealed that IPF lungs are significantly enriched with genes and networks associated with lung development, mainly $\mathrm{WNT} / \beta$-catenin and sonic hedgehog ( $\mathrm{SHH}$ ) signaling pathways ${ }^{29,40}$. 
In IPF lungs, the $\mathrm{WNT} / \beta$-catenin signaling pathway with its main canonical signal transducers, Gsk-3 $\beta$ and $\beta$-catenin, has been detected to be significantly activated mainly in hyperplastic type II epithelial cells, with nuclear translocation of $\beta$-catenin and over-expression of its response genes, like cyclin D1 and matrix metalloprotease-741,42. Likewise, WNT5A has been found significantly increased in IPF fibroblasts, which seem to play a role in fibroblast expansion through non-canonical WNT/ beta-catenin pathway ${ }^{43}$. Moreover, increased WNT signaling pathway components (such as the coreceptor LRP5 and its paralog LRP6) are associated with rapid progression of $\mathrm{IPF}^{44}$.

The $\mathrm{SHH}$ is critical for embryonic lung formation, regulating branching morphogenesis and mesenchymal proliferation ${ }^{45}$. Two recent reports demonstrated the upregulation of $\mathrm{SHH}$ in epithelial cells lining fibrotic areas in IPF lungs while it was undetectable in normal lungs ${ }^{46,47}$. Abnormal expression of the receptor patched, the signal transducer smoothened, and the transcription factor GLI1 in either epithelial cells or fibroblasts was also revealed in IPF lungs. In these studies it was demonstrated that $\mathrm{SHH}$ induces fibroblast migration, proliferation, and resistance to apoptosis, and that it has a profibrotic cross-talk with TGF- $\beta 1$. Actually, smoothened was required for TGF$\beta 1$-induced myofibroblastic differentiation, while GLI-dependent transcription in the nucleus was required for the TGF- $\beta 1$ effects on normal and IPF fibroblasts during differentiation to myofibroblasts ${ }^{47}$. There is additional evidence for interactions between TGF- $\beta 1$ and Wnt/b-catenin pathways during the development of IPF ${ }^{48}$. The TGF- $\beta$-induced convergence of $\beta$-catenin-dependent and canonical Smad3 signaling is critical during TGF- $\beta$-induced epithelial to mesenchymal transition, linking TGF- $\beta /$ Smad3 to pleiotropic $\beta$-catenin/CBP-dependent signaling pathways.

\section{Fibroblasts and the scarring formation}

Fibroblast migration, proliferation, and activation through myofibroblast differentiation play a central role in the abnormal architectural remodeling of IPF. The source of fibroblasts in this disease is unclear, but there is evidence indicating that they may arise from interstitial resident fibroblasts, epithelial to mesenchymal transition, and from the differentiation of recruited circulating bone marrow-derived fibrocytes ${ }^{2,49}$. More recently it has been proposed that Foxd1 progenitor-derived pericytes are also an important source of lung myofibroblasts ${ }^{50}$. However, the real contribution of any, some, or all of these putative sources in IPF is largely unknown.

Activated myofibroblasts, characterized particularly for the formation of stress fibers and expression of $\alpha$-smooth muscle actin are key effector cells in IPF and other fibrotic disorders ${ }^{51}$. They produce large amounts of dense collagens and other components of the ECM and also can maintain a contractile force over long periods of time. As matrix accumulates, it becomes rigid, and the stiffness of the scar tissue has a strong influence on myofibroblast differentiation and progression of fibrosis. Actually, mechanical stress is one of the most potent factors controlling myofibroblast fate and development ${ }^{51}$. Also important is that ECM is a reservoir for a wide range of growth factors including TGF-b and connective tissue growth factor that may be released through 
ECM remodeling in the fibrogenic microenvironment, perpetuating the fibrotic response.

\section{EMERGING THERAPEUTIC OPTIONS}

Idiopathic pulmonary fibrosis is a progressive and incapacitating respiratory condition for which there is no cure. For decades, IPF patients were treated with varying doses/duration of corticosteroids and subsequently with the combination of prednisone plus some immunosuppressive drugs such as azathioprine or cyclophosphamide ${ }^{52}$. Ten years ago, $\mathrm{N}$-acetyl-cysteine (NAC) combined with prednisone and azathioprine was introduced to the treatment of IPF and this triple therapy became a standard of care worldwide for a few years. However, this triple therapy was demonstrated to be harmful to patients according to the results of the PANTHER-IPF clinical trial ${ }^{53}$.

In the last decade, several clinical trials using different compounds, including interferon-gamma, endothelin receptor antagonists (bosentan, macitentan, ambrisentan), anticoagulants (warfarin), a modified p75 receptor of tumor necrosis factor that inhibits its action (etanercept), a tyrosine kinase inhibitor (imatinib), and NAC monotherapy, also showed disappointing results ${ }^{52}$.

Recently, and after several placebo-controlled clinical trials, pirfenidone and nintedanib were the first drugs showing some treatment benefit in patients with IPF. Pirfenidone appears to have pleiotropic antifibrotic and anti-inflammatory effects in experimental and cell-based models, but its mechanism of action is unknown. Nintedanib is a tyrosine kinase inhibitor that targets the platelet-derived growth factor receptors $\alpha / \beta$, fibroblast growth factor receptors $1-3$, and vascular endothelial growth factor receptors 1-3, some of them implicated in fibroblast migration, proliferation, and activation.

Both drugs decreased the decline in forced vital capacity (FVC) compared with the placebo group and also revealed a numerical trend toward improvement in mortality (hazard ratios $<1)^{54-59}$. It is important to note, however, that this effect was observed after a relatively short time ( 1 year), and in this context, long-term studies are needed to evaluate whether these drugs will slow the disease process for a longer duration, and importantly, if these positive effects will also be seen in patients with severe functional impairment and/or comorbidities. Both agents were reasonably well tolerated. Gastrointestinal and skin problems were the most frequent adverse events with pirfenidone, and gastrointestinal adverse effects (mainly diarrhea) were most common with nintedanib.

There is some controversy about the treatment of gastroesophageal reflux (GER) in IPF. There are currently no controlled studies establishing the efficacy of anti-GER treatment in slowing disease progression. Interestingly, a recent meta-analysis of the placebo arms of three IPF clinical trials revealed that patients receiving antacid therapy showed a significantly slower decline in $\mathrm{FVC}^{60}$. Also, some studies comparing outcomes in patients on and off proton pump inhibitor therapy have suggested that this therapy seems to be associated with reduced rates of acute exacerbations ${ }^{61}$.

Lung transplantation represents a therapeutic option in IPF patients refractory to medical management, and there is some evidence indicating that double-lung transplants may 
result in better survival than single-lung transplants. In a recent exploratory study, it was demonstrated that after confounders were controlled for propensity score analysis, double-lung transplants $(n=2,124)$ were associated with better graft survival in patients with IPF than single-lung transplants $(n=2,010)$. The adjusted median survival was 65.2 vs. 50.4 months $(\mathrm{p}<0.001)^{62}$.

Non-pharmacological therapies for IPF include pulmonary rehabilitation and occupational therapy to provide advice on energy conservation in activities of daily living and stress management, and ambulatory oxygen to improve exercise capacity and to facilitate activities of daily living in order to improve quality of life ${ }^{63}$. Oxygen should be indicated for exercise (with a six-minute walk showing the magnitude of exercise-induced desaturation and indicating the required level of oxygen supplementation) and during sleep because nocturnal hypoxemia is common and may impact on quality of life 63 .

\section{REFERENCES}

1. Raghu G, Collard HR, Egan JJ, et al. ATS/ERS/JRS/ALAT Committee on Idiopathic Pulmonary Fibrosis. An official ATS/ERS/JRS/ALAT statement: idiopathic pulmonary fibrosis: evidence-based guidelines for diagnosis and management. Am J Respir Crit Care Med. 2011;183:788-824.

2. King TE, Pardo A, Selman M. Idiopathic pulmonary fibrosis. Lancet. 2011;378:1949-61.

3. Loveman E, Copley VR, Colquitt J, et al. The clinical effectiveness and cost-effectiveness of treatments for idiopathic pulmonary fibrosis: a systematic review and economic evaluation. Health Technol Assess. 2015;19:1-336.

4. Selman M, Carrillo G, Estrada A, et al. Accelerated variant of idiopathic pulmonary fibrosis: clinical behavior and gene expression pattern. PLoS One. 2007;2:e482.

5. Collard HR, Moore BB, Flaherty KR, et al. Idiopathic Pulmonary Fibrosis Clinical Research Network Investigators. Acute exacerbations of idiopathic pulmonary fibrosis. Am J Respir Crit Care Med. 2007;176:636-43.

6. Selman M, Pardo A. Revealing the pathogenic and aging-related mechanisms of the enigmatic idiopathic pulmonary fibrosis. An integral model. Am J Respir Crit Care Med. 2014;189:1161-72.

7. Armanios M. Telomerase and idiopathic pulmonary fibrosis. Mutat Res. 2012;730:52-8.
8. Steele MP, Schwartz DA. Molecular mechanisms in progressive idiopathic pulmonary fibrosis. Annu Rev Med. 2013;64:265-76.

9. Seibold MA, Wise AL, Speer MC, et al. A common MUC5B promoter polymorphism and pulmonary fibrosis. N Engl J Med. 2011;364:1503-12.

10. Borie R, Crestani B, Dieude P, et al. The MUC5B variant is associated with idiopathic pulmonary fibrosis but not with systemic sclerosis interstitial lung disease in the European Caucasian population. PLoS One. 2013;8:e70621.

11. Peljto AL, Selman M, Kim DS, et al. The MUC5B promoter polymorphism is associated with idiopathic pulmonary fibrosis in a Mexican cohort but is rare among Asian ancestries. Chest. 2015;147:460-4.

12. Peljto AL, Zhang Y, Fingerlin TE, et al. Association between the MUC5B promoter polymorphism and survival in patients with idiopathic pulmonary fibrosis. JAMA. 2013;309:2232-9.

13. Mushiroda T, Wattanapokayakit S, Takahashi A, et al. Pirfenidone Clinical Study Group. A genome-wide association study identifies an association of a common variant in TERT with susceptibility to idiopathic pulmonary fibrosis. J Med Genet. 2008;45:654-6.

14. Fingerlin TE, Murphy E, Zhang W, et al. Genome-wide association study identifies multiple susceptibility loci for pulmonary fibrosis. Nat Genet. 2013;45:613-20.

15. Noth I, Zhang Y, Ma SF, et al. Genetic variants associated with idiopathic pulmonary fibrosis susceptibility and mortality: a genome-wide association study. Lancet Respir Med. 2013;1:309-17.

16. Zhu L, Wang L, Luo X, et al. Tollip, an intracellular trafficking protein, is a novel modulator of the transforming growth factor- $\beta$ signaling pathway. J Biol Chem. 2012;287:39653-6.

17. Raghu G, Weycker D, Edelsberg J, Bradford WZ, Oster G. Incidence and prevalence of idiopathic pulmonary fibrosis. Am J Respir Crit Care Med. 2006;174:810-16.

18. López-Otín C1, Blasco MA, Partridge L, Serrano M, Kroemer G. The hallmarks of aging. Cell. 2013;153:1194-217.

19. Vassilakis DA, Sourvinos G, Spandidos DA, Siafakas NM, Bouros D. Frequent genetic alterations at the microsatellite level in cytologic sputum samples of patients with idiopathic pulmonary fibrosis. Am J Respir Crit Care Med. 2000;162:1115-19.

20. Alder JK, Chen JJ, Lancaster L, et al. Short telomeres are a risk factor for idiopathic pulmonary fibrosis. Proc Natl Acad Sci USA. 2008;105:13051-6.

21. Cronkhite JT, Xing C, Raghu G, et al. Telomere shortening in familial and sporadic pulmonary fibrosis. Am J Respir Crit Care Med. 2008;178:729-37.

22. Bueno M, Lai YC, Romero Y, et al. PINK1 deficiency impairs mitochondrial homeostasis and promotes lung fibrosis. J Clin Invest. 2015;125:521-38.

23. Jain M, Rivera S, Monclus EA, et al. Mitochondrial reactive oxygen species regulate transforming growth factor-beta signaling. J Biol Chem. 2013;288:770-7.

24. Minagawa S, Araya J, Numata T, et al. Accelerated epithelial cell senescence in IPF and the inhibitory role of SIRT6 in TGF- $\beta$-induced senescence of human bronchial epithelial cells. Am J Physiol Lung Cell Mol Physiol 2011;300:L391-401.

25. Araya J, Kojima J, Takasaka N, et al. Insufficient autophagy in idiopathic pulmonary fibrosis. Am J Physiol Lung Cell Mol Physiol. 2013;304:L56-69.

26. Tanjore H, Blackwell TS, Lawson WE. Emerging evidence for endoplasmic reticulum stress in the pathogenesis of idiopathic pulmonary fibrosis. Am J Physiol Lung Cell Mol Physiol. 2012;302:L721-9.

27. Patel AS, Lin L, Geyer A, et al. Autophagy in idiopathic pulmonary fibrosis PLoS One. 2012;7:e41394.

28. Issa JP. Aging and epigenetic drift: a vicious cycle. J Clin Invest. 2014;124:24-9.

29. Yang IV, Pedersen BS, Rabinovich E, et al. Relationship of DNA methylation and gene expression in idiopathic pulmonary fibrosis. Am J Respir Cri Care Med. 2014;190:1263-72.

30. Mousseau DD, Chapelsky S, De Crescenzo G, et al. A direct interaction between transforming growth factor (TGF)-betas and amyloid-beta protein affects fibrillogenesis in a TGF-beta receptor-independent manner. J Biol Chem. 2003;278:38715-22.

31. Cheedipudi S, Genolet O, Dobreva G. Epigenetic inheritance of cell fates during embryonic development. Front Genet. 2014;5:19. 
32. Wilborn J, Crofford LJ, Burdick MD, Kunkel SL, Strieter RM, Peters-Golden M. Cultured lung fibroblasts isolated from patients with idiopathic pulmonary fibrosis have a diminished capacity to synthesize prostaglandin E2 and to express cyclooxygenase-2. J Clin Invest. 1995;95:1861-8.

33. Coward WR, Watts K, Feghali-Bostwick CA, Knox A, Pang L. Defective histone acetylation is responsible for the diminished expression of cyclooxygenase 2 in idiopathic pulmonary fibrosis. Mol Cell Biol. 2009;29:4325-39.

34. Tay Y, Rinn J, Pandolfi PP. The multilayered complexity of ceRNA crosstalk and competition. Nature. 2014;505:344-52.

35. Vettori S, Gay S, Distler O. Role of microRNAs in fibrosis. Open Rheumatol J. 2012;6:130-9.

36. Pandit KV, Milosevic J. MicroRNA regulatory networks in idiopathic pulmonary fibrosis. Biochem Cell Biol. 2015;5:1-9.

37. Milosevic J, Pandit K, Magister M, et al. Profibrotic role of miR-154 in pulmonary fibrosis. Am J Respir Cell Mol Biol. 2012;47:879-87.

38. Parker MW, Rossi D, Peterson M, et al. Fibrotic extracellular matrix activates a profibrotic positive feedback loop. J Clin Invest. 2014;124:1622-35.

39. Berschneider B, Ellwanger DC, Baarsma HA, et al. miR-92a regulates TGF$\beta 1$-induced WISP1 expression in pulmonary fibrosis. Int J Biochem Cell Biol. 2014;53:432-41.

40. Selman M, Pardo A, Kaminski N. Idiopathic pulmonary fibrosis: aberrant recapitulation of developmental programs? PLoS Med. 2008;5:e62.

41. Chilosi M, Poletti V, Zamo A, et al. Aberrant Wnt/beta-catenin pathway activation in idiopathic pulmonary fibrosis. Am J Pathol. 2003;162:1495-502.

42. Königshoff M, Balsara N, Pfaff EM, et al. Functional Wnt signaling is increased in idiopathic pulmonary fibrosis. PLoS One. 2008;3:e2142.

43. Vuga LJ, Ben-Yehudah A, Kovkarova-Naumovski E, et al. WNT5A is a regulator of fibroblast proliferation and resistance to apoptosis. Am J Respir Cell Mol Biol. 2009;41:583-9.

44. Lam AP, Herazo-Maya JD, Sennello JA, et al. Wnt coreceptor Lrp5 is a driver of idiopathic pulmonary fibrosis. Am J Respir Crit Care Med. 2014;190:185-95.

45. Kugler MC, Joyner AL, Loomis CA, Munger JS. Sonic hedgehog signaling in the lung. From development to disease. Am J Respir Cell Mol Biol. 2015;52:1-13.

46. Bolaños AL, Milla CM, Lira JC, et al. Role of Sonic Hedgehog in idiopathic pulmonary fibrosis. Am J Physiol Lung Cell Mol Physiol. 2012;303:L978-90.

47. Cigna N, Farrokhi Moshai E, Brayer S, et al. The hedgehog system machinery controls transforming growth factor- $\beta$-dependent myofibroblastic differentiation in humans: involvement in idiopathic pulmonary fibrosis. Am J Pathol. 2012;181:2126-37.

48. Zhou B, Liu Y, Kahn M, et al. Interactions between $\beta$-catenin and transforming growth factor- $\beta$ signaling pathways mediate epithelial-mesenchymal transition and are dependent on the transcriptional co-activator cAMP-response element-binding protein (CREB)-binding protein (CBP). J Biol Chem. 2012;287:7026-38.
49. Andersson-Sjöland A, de Alba CG, Nihlberg K, et al. Fibrocytes are a potential source of lung fibroblasts in idiopathic pulmonary fibrosis. Int J Biochem Cell Biol. 2008;40:2129-40.

50. Hung C, Linn G, Chow YH, et al. Role of lung pericytes and resident fibroblasts in the pathogenesis of pulmonary fibrosis. Am J Respir Crit Care Med. 2013;188:820-30.

51. Hinz B. Mechanical aspects of lung fibrosis: a spotlight on the myofibroblast. Proc Am Thorac Soc. 2012;9:137-47.

52. Raghu G, Selman M. Nintedanib and pirfenidone. New antifibrotic treat ments indicated for idiopathic pulmonary fibrosis offer hopes and raises questions. Am J Respir Crit Care Med. 2015;191:252-4.

53. Raghu G, Anstrom KJ, King TE, Lasky JA, Martinez FJ. Idiopathic Pulmonary Fibrosis Clinical Research Network. Prednisone, azathioprine, and $\mathrm{N}$-acetylcysteine for pulmonary fibrosis. N Engl J Med. 2012;366:1968-77.

54. Taniguchi H, Ebina M, Kondoh Y, et al. Pirfenidone Clinical Study Group in Japan. Pirfenidone in idiopathic pulmonary fibrosis. Eur Respir J 2010;35:821-9.

55. Noble PW, Albera C, Bradford WZ, et al. CAPACITY Study Group. Pirfenidone in patients with idiopathic pulmonary fibrosis (CAPACITY): two randomised trials. Lancet. 2011;377:1760-9.

56. King TE, Bradford WZ, Castro-Bernardini S, et al. ASCEND Study Group. A phase 3 trial of pirfenidone in patients with idiopathic pulmonary fibrosis. N Engl J Med. 2014;370:2083-92.

57. Richeldi L, Costabel U, Selman M, et al. Efficacy of a tyrosine kinase inhibitor in idiopathic pulmonary fibrosis. N Engl J Med. 2011;365:1079-87.

58. Richeldi L, du Bois RM, Raghu G, et al. INPULSIS Trial Investigators Efficacy and safety of nintedanib in idiopathic pulmonary fibrosis. N Eng J Med. 2014;370:2071-82.

59. Karimi-Shah BA, Chowdhury BA. Forced vital capacity in idiopathic pulmonary fibrosis-FDA review of pirfenidone and nintedanib. N Engl J Med. 2015;372:1189-91.

60. Lee JS, Collard HR, Anstrom KJ, et al. IPFnet Investigators. Anti-acid treat ment and disease progression in idiopathic pulmonary fibrosis: an analysis of data from three randomised controlled trials. Lancet Respir Med. 2013;1:369-76.

61. Johannson K, Collard HR. Acute exacerbation of idiopathic pulmonary fibrosis: A proposal. Curr Respir Care Rep. 2013;2:10.

62. Schaffer JM, Singh SK, Reitz BA, Zamanian RT, Mallidi HR. Single-vs double-lung transplantation in patients with chronic obstructive pulmonary disease and idiopathic pulmonary fibrosis since the implementation of lung allocation based on medical need. JAMA. 2015;313:936-48.

63. Thickett DR, Kendall C, Spencer LG, et al. Improving care for patients with idiopathic pulmonary fibrosis (IPF) in the UK: a round table discussion Thorax. 2014:69:1136-40. 